\title{
On the Existence and Symmetry Properties of Finite Total Mass Solutions of the Matukuma Equation, the Eddington Equation and their Generalizations
}

\author{
Yi Li \& WeI-Ming NI
}

\section{§ 1. Introduction and Statements of Main Results}

In 1915 A. S. EDDINGTON introduced the following equation as a mathematical model to describe the dynamics of globular clusters of stars ([E]):

$$
\Delta u+\frac{e^{2 u}}{1+|x|^{2}}=0 \quad \text { in } \mathbb{R}^{3}
$$

where $u>0$ represents the gravitational potential, $\varrho=-\frac{1}{4 \pi} \Delta u=\frac{1}{4 \pi\left(1+|x|^{2}\right)} e^{2 u}$ is the density and $\int_{\mathbb{R}^{3}} \varrho(x) d x$ represents the total mass. Fifteen years later, in 1930 , on the basis of his physical intuition, T. Matukuma proposed the following equation to improve EDDINGTON's model ([M]):

$$
\Delta u+\frac{1}{1+|x|^{2}} u^{p}=0 \quad \text { in } \mathbb{R}^{3}
$$

where $p>1$ and again $u>0$ is the gravitational potential with $\int_{\mathbb{R}^{3}} \frac{1}{4 \pi\left(1+|x|^{2}\right)} u^{p} d x$ representing the total mass. Since the physical models are spherically symmetric, positive radial entire solutions of (1.1) or (1.2) are of particular interest, and in this case equations (1.1) and (1.2) reduce to the following ordinary differential equations, respectively

$$
\begin{gathered}
u_{r r}+\frac{2}{r} u_{r}+\frac{1}{1+r^{2}} e^{2 u}=0 \quad \text { in }[0, \infty), \\
u(0)=\alpha, \quad u_{r}(0)=0
\end{gathered}
$$


and

$$
\begin{gathered}
u_{r r}+\frac{2}{r} u_{r}+\frac{1}{1+r^{2}} u^{p}=0 \quad \text { in }[0, \infty), \\
u(0)=\alpha, \quad u_{r}(0)=0,
\end{gathered}
$$

where $\alpha>0$. For each $\alpha>0$, we shall denote the (global) unique solution of $\left(1.1_{\alpha}\right)$ (or $\left.\left(1.2_{\alpha}\right)\right)$ by $u=u(r ; \alpha)$. Studying the structure of solutions of $\left(1.2_{\alpha}\right)$, MATUKUMA conjectured that

(i) if $p<3$, then $u(r ; \alpha)$ has a finite zero for every $\alpha>0$,

(ii) if $p=3$, then $u(r ; \alpha)$ is a positive entire solution with finite total mass for every $\alpha>0$,

(iii) if $p>3$, then $u(r ; \alpha)$ is a positive entire solution with infinite total mass for every $\alpha>0$.

In 1938 MATUKUMA found an interesting exact solution

$$
u(r ; \sqrt{3})=\left(\frac{3}{1+r^{2}}\right)^{\frac{1}{2}}
$$

for $\left(1.2_{\alpha}\right)$ with $p=3$ which confirms part of his conjecture. Since then, there seems to be very little mathematical contribution in the literature on these two equations until the very recent works of Ni \& YoTSUTANI [NY1], [NY2].

First, it was observed in [NY2] that the Eddington equation (i.e. (1.1)) does not have any positive radial entire solutions (which perhaps indicates that the MATUKUMA equation is indeed a better physical model). Concerning Maturuma's conjecture, Ni \& YotsutanI established the following result which shows that equation (1.2) is perhaps more delicate than MATUKuma had expected.

Theorem $[\mathrm{NY} 1,2]$. Let $u=u(r ; \alpha)$ be the solution of $\left(1.2_{\alpha}\right)$.

(A) If $1<p<5$, then $u(r ; \alpha)$ has a finite zero for every sufficiently large $\alpha>0$.

(B) If $1<p<5$, then $u(r ; \alpha)$ is a positive entire solution with infinite total mass for every sufficiently small $\alpha>0$.

(C) If $p \geqq 5$, then $u(r ; \alpha)$ is a positive entire solution with infinite total mass for every $\alpha>0$.

Note that the existence of positive entire radial solutions with finite total mass is left open in [NY1, 2]. In this paper, using a variational approach, we shall settle this question; that is, we shall prove the following

Theorem 1.1. For every $1<p<5$, there is an $\alpha^{*}>0$ such that the solution $u\left(r ; \alpha^{*}\right)$ of $\left(1.2_{\alpha^{*}}\right)$ is positive in $[0, \infty)$ and has finite total mass, i.e.

$$
\int_{\mathbb{R}^{3}} \frac{1}{1+|x|^{2}} u^{p}\left(|x| ; \alpha^{*}\right) d x<\infty .
$$


Remark 1.1. It is established in [LN] (Theorem 2.41) that a positive radial entire solution $u$ of (1.2) in $\mathbb{R}^{3}$ must satisfy either

$$
u(r) \sim r^{-1}
$$

or

$$
u(r) \sim(\log r)^{\frac{1}{1-p}}
$$

at $\infty$. (Here the notation " $f \sim g$ at $\infty$ " means that there are two positive constants $C_{1}, C_{2}$ such that $C_{1} g \geqq f \geqq C_{2} g$ at $\infty$.) It is then easy to verify that a radial finite total mass solution of (1.2) must satisfy (1.4) and a radial entire solution of (1.2) which has infinite total mass must satisfy (1.5) at $\infty$. Thus we conclude from Lemmas 7.1 and 7.2 in [NY2] that a radial finite total mass solution $u$ of (1.2) must satisfy

$$
\lim _{r \rightarrow \infty} r u(r)=\frac{1}{4 \pi} \int_{\mathbb{R}^{3}} \frac{1}{1+|x|^{2}} u^{p}(x) d x
$$

i.e. the limit of $|x| u(x)$ at $\infty$ is exactly the total mass of $u$.

Up to this point, we have only studied radial solutions of equations (1.1) and (1.2). It is nonetheless an interesting and natural mathematical question whether equation (1.2) possesses only positive radial entire solutions. To investigate this problem we apply the arguments used in $[\mathrm{GNN}]$ to the following equation

$$
\Delta u+f(r, u)=0 \quad \text { in } \mathbb{R}^{n},
$$

where $n \geq 3, r=|x|$ and $f$ is a continuous function which is strictly decreasing in $r \geqq 0$ and nondecreasing in $u>0$. The starting point here is a refinement of Theorem 1", p. 380 in [GNN], which is established in the Appendix (Theorem A.1). Using that we are able to prove, for instance, the following

Proposition 1.1. Suppose that $u$ is a positive $C^{2}$ solution of (1.6) which tends to zero at infinity. Assume that there exist $p>0, \tau<-2$ and $C>0$ such that

$$
0 \leqq f(r, t) \leqq C\left(1+r^{n}\right)^{\frac{\tau}{n}} t^{p}
$$

holds in $[0, \infty) \times\left[0,\|u\|_{L^{\infty}\left(\mathrm{R}^{\eta}\right)}\right]$. If $p(n-2)>n+1+\tau$, then $u$ must be radially symmetric about the origin and $u_{r}<0$ for $r>0$. Furthermore,

$$
\lim _{r \rightarrow \infty} r^{n-2} u(r)=k>0 \text {. }
$$

Proposition 1.2. Every bounded positive solution $u$ of (1.6) is radially symmetric about the origin and $u_{r}<0$ in $r>0$ if

$$
0 \leqq f(r, t) \leqq C(t)\left(1+r^{n}\right)^{\frac{\tau}{n}}
$$

holds in $\mathbb{R}^{+} \times \mathbb{R}^{+}$for some $\tau<-(n+1)$ and $C(t)$ is nondecreasing in $\mathbb{R}^{+}$.

The novelty here seems to be that no decay rate on solutions is required. In fact, $u$ does not even have to tend to zero at $\infty$ in the second proposition. 
We shall also apply Theorem A.1 to equation (1.2) to obtain

Theorem 1.2. Let $2<p<5$. Then every bounded positive entire solution $u$ of equation (1.2) with finite total mass is radially symmetric about the origin and $u_{r}<0$ in $r>0$. Furthermore,

$$
\lim _{r \rightarrow \infty} r u(r)=k>0 .
$$

Theorem 1.3. Let $p \geqq 5$. Then every bounded positive entire solution of (1.2) has infinite total mass.

We should remark that Theorems 1.1, 1.2, 1.3 and Remark 1.1 admit various extensions to $\mathbb{R}^{n}, n \geqq 3$, and to the more general equation

$$
\Delta u+K u^{p}=0 \quad \text { in } \mathbb{R}^{n},
$$

where $K$ is a suitable generalization of $1 /\left(1+r^{2}\right)$. To this connection we ought to mention a recent article of BATT, FALTENBACHER \& HORST [BFH] in which the following model was proposed

$$
\Delta u+\frac{|x|^{\lambda-2}}{\left(1+|x|^{2}\right)^{\lambda / 2}} u^{p}=0 \quad \text { in } \mathbb{R}^{3},
$$

where $\lambda>0, p>1$. Note that when $\lambda=2$, this reduces to Matukuma's model (1.2). Some of our analysis does apply to this more general model. (See Section 2 below.)

We now come to the following generalized Eddington equation

$$
\Delta u+H e^{2 u}=0 \quad \text { in } \mathbb{R}^{n}, n \geqq 3 .
$$

It will be assumed throughout this paper that $H(x) \geqq 0$ in $\mathbb{R}^{n}$ and is locally Hölder continuous. It was already established in [NY2] (Theorems 8.2 and 8.4) and in $] \mathrm{K}],[\mathrm{K} 0]$ that in case $H(x)=H(|x|)$ we have

Theorem (D). (1.11) possesses no positive radial entire solution if

$$
\frac{1}{n-2} \int_{0}^{\infty} r H(r) d r=\infty
$$

(E) (1.11) possesses infinitely many positive radial entire solutions if

$$
\frac{1}{n-2} \int_{0}^{\infty} r H(r) d r<\frac{1}{2 e}
$$

In this paper we shall improve this theorem as well as extend it to non-radial case. First, we present a sufficient condition.

Theorem 1.4. Suppose that

$$
\sup _{x \in \mathbb{R}^{n}} \int_{\mathbb{R}^{n}} \frac{H(y)}{n(n-2) \omega_{n}|x-y|^{n-2}} d y<\frac{1}{2 e},
$$

where $\omega_{n}$ denotes the volume of the unit ball in $\mathbb{R}^{n}$. Then equation (1.11) possesses infinitely many positive entire solutions in $\mathbb{R}^{n}$. 
We should point out that in case $H(x)=H(|x|),(1.14)$ reduces to (1.13) (see Section 3.2 below).

To state our necessary condition, we need to introduce the following notations. For a given function $\phi$ and a given point $x_{0} \in \mathbb{R}^{n}$, we set

$$
\bar{\phi}_{x_{0}}(r)=\frac{1}{n \omega_{n} r^{n-1}} \int_{\left|x-x_{0}\right|=r} \phi(x) d S\left(=\frac{1}{n \omega_{n|\xi|}} \int_{=1} \phi\left(x_{0}+r \xi\right) d S_{\xi}\right),
$$

which is the spherical mean of $\phi$ at $x_{0}$. In case $x_{0}=0$ (the origin), we shall denote $\bar{\phi}_{0}$ by $\bar{\phi}$ for simplicity. If $\phi \geqq 0$, then for every $p>1$, we define

$$
\bar{\phi}_{x_{0}, p}(r)=\left\{\frac{1}{n \omega_{n} r^{n-1}} \int_{\left|x-x_{0}\right|=r} \phi^{\frac{1}{1-p}}(x) d S\right\}^{1-p}
$$

with the obvious convention that $\bar{\phi}_{x_{0}, p}(r)=0$ if the integral in (1.16) is infinite. $((1.15)$ is the usual spherical mean while (1.16) was first used in [N1].)

Theorem 1.5. Suppose that (1.11) possesses at least one positive entire solution. Then the condition

$$
\frac{1}{n-2} \int_{0}^{\infty} r \bar{H}_{x_{0}, p}(r) d r<\frac{1}{2}
$$

must be satisfied for every $x_{0} \in \mathbb{R}^{n}$ and every $p>1$.

The organization of this paper is as follows. Theorems 1.1, 1.2 and 1.3 (and their extensions) are proved in Section 2. In Section 3, we establish Theorems 1.4 and 1.5. The proofs of Propositions 1.1 and 1.2 are included in the Appendix.

\section{§ 2 Matukuma Equation and its Generalizations}

The purpose of this section is to establish the existence of solutions with finite total mass of the Matukuma equation and its various generalizations. We shall treat the existence question in subsection 2.1 and the symmetry property in subsection 2.2 .

\subsection{Existence of Finite Total Mass Solutions}

In this subsection we shall study the following equation

$$
\Delta u+\frac{|x|^{\lambda-2}}{\left(1+|x|^{2}\right)^{\lambda / 2}} u^{p}=0
$$

in $\mathbb{R}^{n}$, where $p>1, \lambda>0$ and $n \geqq 3$. Note that when $\lambda<2$, the coefficient in the nonlinear term is singular at $x=0$.

We first introduce some notations. Let $E$ be the completion of the set of all smooth, radially symmetric functions with compact support in $\mathbb{R}^{n}$ under the 
norm

$$
\|\varphi\|^{2}=\int_{\mathbb{R}^{n}}|D \varphi|^{2} d x
$$

Using a variational approach, we are able to establish the following

Theorem 2.1. For every $\lambda>0$, equation (2.1) possesses a positive entire weak solution $u$ in $E$ with finite total mass, i.e.

$$
\int_{\mathbb{R}^{n}} \frac{|x|^{\lambda-2}}{\left(1+|x|^{2}\right)^{\lambda / 2}} u^{p}(x) d x<\infty,
$$

provided that $p$ satisfies

$$
1<p<1+\frac{2 \lambda}{n-2}
$$

Furthermore,

(i) if $\lambda \geqq 2$, then the solution $u$ is $C^{2}$ and classical,

(ii) if $1 \neq \lambda<2$, then the solution $u \in C^{\lambda}\left(\mathbb{R}^{n}\right) \cap C^{2}\left(\mathbb{R}^{n} \backslash\{0\}\right)$,

(iii) if $\lambda=1$, then the solution $u \in C^{0,1}\left(\mathbb{R}^{n}\right) \cap C^{2}\left(\mathbb{R}^{n} \backslash\{0\}\right)$;

and

$$
\lim _{r \rightarrow 0} r^{n-1} u_{r}(r)=0
$$

In proving this theorem, we recall the following result (Theorem 3.18 in [LN]) concerning the equation

in $\mathbb{R}^{n}$.

$$
A u+K(|x|)|u|^{p-1} u=0
$$

Lemma 2.1. If $K$ is continuous in $\mathbb{R}^{n} \backslash\{0\}$ and satisfies either

$$
\int_{0}^{\infty} K(r) \mid r^{\frac{n-p(n-2)}{2}} d r<\infty
$$

with $K^{+} \neq 0$, or

(b)

$$
\begin{aligned}
& 0<\int_{0}^{\infty} K^{+}(r) r^{\frac{n-p(n-2)}{2}} d r<\infty, \\
& K^{-} \in L^{2 n /[2 n-(n-2)(p+1)]}\left(\mathbb{P}^{n}\right) \quad \text { if } 1<p<\frac{n+2}{n-2},
\end{aligned}
$$

$$
\begin{array}{ll}
K^{-} \in L^{\infty}\left(\mathbb{R}^{n}\right) & \text { if } p=\frac{n+2}{n-2}, \\
K^{-} \equiv 0 & \text { if } p>\frac{n+2}{n-2} .
\end{array}
$$

Then (2.5) possesses at least one nonnegative nontrivial entire weak solution in $E \cap C^{2}\left(\mathbb{R}^{n} \backslash\{0\}\right)$. 
We should remark that although $K$ was assumed to be locally Hölder continuous in $\mathbb{R}^{n}$ in [LN], the arguments there can be carried over here without any change.

We are now ready for the

Proof of Theorem 2.1. Setting

$$
K(r)=\frac{r^{\lambda-2}}{\left(1+r^{2}\right)^{\lambda / 2}}, \quad \lambda>0,
$$

we see that $K$ satisfies the integral conditions in Lemma 2.1 if and only if (2.3) holds. Therefore, by Lemma 2.1 , there exists a nonnegative nontrivial entire weak solution $u$ of $(2.1)$ in $C^{2}\left(\mathbb{R}^{n} \backslash\{0\}\right)$. The regularity properties of $u$ at $r=0$ (i.e. (i), (ii), (iii) and (2.4) in Theorem 2.1) follow from the arguments in p. 238 in [N2]. (See also Section 4 in [NY2].)

It remains to show that the solution $u$ obtained by Lemma 2.1 above has finite total mass. First, observe that there exists a constant $C>0$ such that

$$
u(r) \leqq C r^{(2-n) / 2} \quad \text { for all } r>0
$$

since $u \in E$ (see e.g. [BL; p. 340]). Next we assert that

$$
u(r) \leqq C r^{2-n} \quad \text { at } r=\infty .
$$

For, by equation (2.1) and the fact that $u \in C^{2}$ for $r>0$ we have

$$
\left(r^{n-1} u_{r}\right)_{r}+r^{n-1} K(r) u^{p}=0 \quad \text { in } r>0 .
$$

Integrating from $\varepsilon$ to $r$ and letting $\varepsilon \rightarrow 0$, we obtain by (2.4)

$$
u_{r}(r)=-r^{1-n} \int_{0}^{r} K(s) u^{p}(s) s^{n-1} d s .
$$

Integrating (2.8) from $r$ to $\infty$ and using the fact that $u(r) \rightarrow 0$ as $r \rightarrow \infty$ (by (2.6)), we have

$$
\begin{aligned}
u(r) & =\int_{r}^{\infty} t^{1-n} \int_{0}^{t} K(s) u^{p}(s) s^{n-1} d s d t \\
& =\frac{1}{n-2} r^{2-n} \int_{0}^{r} \frac{s^{n-3+\lambda}}{\left(1+s^{2}\right)^{\lambda / 2}} u^{p}(s) d s+\frac{1}{n-2} \int_{r}^{\infty} \frac{s^{\lambda-1}}{\left(1+s^{2}\right)^{\lambda / 2}} u^{p}(s) d s,
\end{aligned}
$$

by changing the order of integration. For $r \geqq 1$, it then follows that

$$
u(r) \leqq C\left(r^{2-n}+r^{2-n} \int_{1}^{r} s^{n-3} u^{p}(s) d s+\int_{r}^{\infty} s^{-1} u^{p}(s) d s\right) .
$$

Combining (2.6) and (2.9), we conclude that for $r \geqq 1$

$$
u(r) \leqq C\left(r^{2-n}+r^{p(2-n) / 2}\right) .
$$


Since $p>1$, there is a positive integer $k$ such that

$$
\left(\frac{n-2}{2}\right) p^{k}<n-2 \leqq\left(\frac{n-2}{2}\right) p^{k+1} .
$$

Now substituting (2.10) into (2.9) we obtain

$$
u(r) \leqq C\left(r^{2-n}+r^{\left.p^{2(2-n) / 2}\right)} \quad \text { for } r \geqq 1 .\right.
$$

Iterating this step $k$ times, we arrive at

$$
u(r) \leqq C\left(r^{2-n}+r^{p^{k+1}(2-n) / 2}\right) \leqq C r^{2-n} \quad \text { for } r \geqq 1 .
$$

This proves our assertion (2.7).

It is easily seen from (2.7) and (2.3) that

$$
K(r) u^{p}(r)=O\left(r^{-n-(p-1)(n-2)}\right) \quad \text { at } r=\infty,
$$

and at $r=0$ we have $K(r) u^{p}(r)=O\left(r^{\lambda-2}\right)$. Thus (2.2) holds and the solution $u$ has finite total mass. Q.E.D.

Corollary 2.1. Let $u=u(r ; \alpha)$ denote the solution of the following initial value problem

$$
\begin{gathered}
u_{r r}+\frac{n-1}{r} u_{r}+\frac{r^{\lambda-2}}{\left(1+r^{2}\right)^{1 / 2}} u_{+}^{p}=0, \quad r>0, \\
u_{r}(0)=0, \quad u(0)=\alpha>0,
\end{gathered}
$$

where $\lambda \geqq 2, n \geqq 3,1<p<1+2 \lambda /(n-2)$ and $u^{+} \equiv \max \{u, 0\}$. Then

(i) for every $\alpha>0, u(r ; \alpha)$ is a $C^{2}$ solution in the entire ray $r \geqq 0$,

(ii) for every large $\alpha>0, u(r ; \alpha)$ has a finite zero,

(iii) for every small $\alpha>0, u(r ; \alpha)$ is a positive entire solution which satisfies

$$
0<\liminf _{r \rightarrow \infty}(\log r)^{\frac{1}{p-1}} u(r) \leqq \limsup _{r \rightarrow \infty}(\log r)^{\frac{1}{p-1}} u(r)<\infty,
$$

and thus has infinite total mass,

(iv) there exists at least one $\alpha^{*}>0$ such that $u\left(r ; \alpha^{*}\right)$ is a positive entire solution with

$$
\limsup _{r \rightarrow \infty} r^{n-2} u(r)=c \in(0, \infty)
$$

and thus has finite total mass.

Proof. (i) is standard (see e.g. Proposition 4.2 in [NY2]). (ii) is proved in Corollary 5.1, [NY2]. (iii) follows from Theorem 7.1 in [NY2] (where the conditions (K.1), (K.4) and (K.5) are easily verified) and Theorem 2.41 in [LN] (which in particular guarantees (2.12)). The existence in (iv) follows from Theorem 2.1 above while (2.13) follows from Lemma 7.1 in [NY2]. Q.E.D. 


\subsection{Symmetry Properties of Solutions having Finite Total Mass}

This subsection is devoted to the study of the symmetries of finite total mass solutions of the following equation

$$
\begin{gathered}
\Delta u+K(x) u^{p}=0 \quad \text { in } \mathbb{R}^{n}, \\
u>0 \quad \text { in } \mathbb{R}^{n},
\end{gathered}
$$

where $p>0$ and $n \geqq 3$. Here a solution $u$ of (2.14) is said to have finite total mass if $K u^{p} \in L^{1}\left(\mathbb{R}^{n}\right)$. We shall assume throughout this subsection that $K(x)$ is locally Hölder-continuous on $\mathbb{R}^{n}$.

First, we derive the asymptotic behavior of such solutions.

Lemma 2.2. Let

$$
v(x) \equiv \int_{\mathbb{R}^{n}} \frac{\tilde{K}(y)}{c|x-y|^{n-2}} d y, \quad x \in \mathbb{R}^{n},
$$

where $c=n(n-2) \omega_{n}, \tilde{K}$ is locally Hölder continuous with $\tilde{K} \in L^{1}\left(\mathbb{R}^{n}\right) \cap L^{\infty}\left(\mathbb{R}^{n}\right)$ and $\tilde{K}(x)=O\left(|x|^{\tau}\right)$ at $\infty$ for some $\tau \leqq 0$. Then $\Delta v+\tilde{K}=0$ in $\mathbb{R}^{n}$. Furthermore, we have

$$
v(x)=\left\{\begin{array}{lll}
o\left(|x|^{\frac{n-2}{n} \tau}\right) & \text { at } x=\infty & \text { if } \tau>-n, \\
O\left(|x|^{2-n}\right) & \text { at } x=\infty & \text { if } \tau \leqq-n
\end{array}\right.
$$

Proof. First observe that $v(x)$ is well defined and it follows from standard arguments that $\Delta v+\tilde{K}=0$ in $\mathbb{R}^{n}$ (see e.g. Lemma 2.8 in [LN]).

It remains to show that $(2.15)$ holds. Without loss of generality, we may assume that $0 \geqq \tau \geqq-n$. For fixed $\delta>0$, we have

$$
\begin{aligned}
& |v(x)| \leqq C \int_{\mathrm{R}^{n}} \frac{|\tilde{K}(y)|}{|z-y|^{n-2}} d y
\end{aligned}
$$

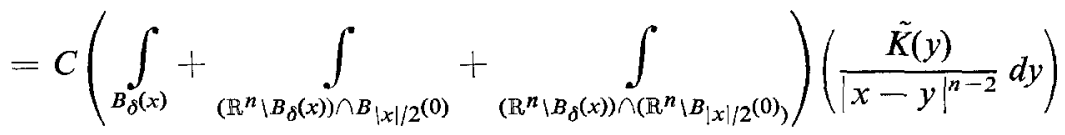

$$
\begin{aligned}
& \leqq C\left\{\|K\|_{L^{\infty}\left(B_{\delta}(x)\right)} \int_{0}^{\delta} r d r+|x|^{2-n} \int_{[|y|<|x| / 2]}|\tilde{K}(y)| d y+\delta^{2-n} \int_{[|y|>|x| / 2]}|\tilde{K}(y)| d y\right\} \\
& \leqq C\left\{\delta^{2}\|\tilde{K}\|_{L^{\infty}\left(B_{\delta}(x)\right)}+|x|^{2-n}\|\tilde{K}\|_{L^{1}\left(\mathbb{R}^{n}\right)}+\delta^{2-n} \int_{[|y|>|x| / 2]}|\tilde{K}(y)| d y\right\} .
\end{aligned}
$$

Setting

$$
\delta=\left\{|x|^{-\tau} \int_{[\mid y]>\{x \mid / 2]}|\tilde{K}(y)| d y\right\}^{\frac{1}{n}}
$$


we have $\delta<|x| / 2$ for large $|x|$ since $-\tau \leqq n$ and $\tilde{K} \in L^{1}\left(\mathbb{R}^{n}\right)$. Thus

$$
\|\tilde{K}\|_{L^{\infty}\left(B_{\delta}(x)\right)}=O\left(|x|^{\tau}\right)
$$

for large $|x|$. It then follows that, for $|x|$ large,

$$
|v(x)| \leqq C\left\{|x|^{\frac{n-2}{n} \tau}\left(\int_{[|y|>|x| / 2]}|\tilde{K}(y)| d y\right)^{\frac{2}{n}}+|x|^{2-n}\|\tilde{K}\|_{L^{1}\left(\mathbb{R}^{n}\right)}\right\} .
$$

This proves (2.15) since

$$
\lim _{x \rightarrow \infty} \int_{[|y|>|x| / 2]}|\tilde{K}(y)| d y=0 .
$$

Q.E.D.

Lemma 2.3. Let $u$ be a bounded positive solution of (2.14) with $\lim _{x \rightarrow \infty} \inf u(x)=0$. Suppose that $K u^{p} \in L^{1}\left(\mathbb{R}^{n}\right) \cap L^{\infty}\left(\mathbb{R}^{n}\right)$ and $K(x)=O\left(|x|^{\tau}\right)$ at $\infty$ for some $\tau \leqq 0$. Then

$$
u(x)=\int_{\mathbb{R}^{n}} \frac{K(y) u^{p}(y)}{c|x-y|^{n-2}} d y
$$

Furthermore, if $\tau<0$ then for any $\varepsilon>0$

$$
u(x)=\left\{\begin{array}{llll}
O\left(\left|x^{2-n}\right|\right) & \text { at } x=\infty & \text { if } & p>\frac{n+\tau}{n-2}, \\
O\left(|x|^{\frac{(n-2) \tau}{n-(n-2) p}+\varepsilon}\right) & \text { at } x=\infty & \text { if } & 0<p \leqq \frac{n+\tau}{n-2} .
\end{array}\right.
$$

Proof. Setting $\tilde{K}(x) \equiv K(x) u^{p}(x)$, we see that $\tilde{K} \in L^{1}\left(\mathbb{R}^{n}\right) \cap L^{\infty}\left(\mathbb{R}^{n}\right)$ and $\tilde{K}(x)=$ $O\left(|x|^{\tau}\right)$ for some $\tau \leqq 0$ at $\infty$. Let

$$
v(x)=\int_{\mathbb{R}^{n}} \frac{\tilde{K}(y)}{c|x-y|^{n-2}} d y .
$$

By Lemma 2.2 we have $\Delta(u-v)=0$ and, both $u$ and $v$ are bounded. Thus the Liouville theorem implies that $u-v \equiv$ constant. Since $v \rightarrow 0$ at $\infty$ and $\liminf _{x \rightarrow \infty} u(x)=0$, we conclude that $u \equiv v$ and (2.17) holds.

To prove (2.18) we assume that $0>\tau>-n$. (For otherwise, (2.18) follows directly from (2.15).) It follows from (2.15) that

$$
\tilde{K}(x)=O\left(|x|^{\tau\left(1+\frac{n-2}{n} p\right)}\right) \quad \text { at } x=\infty .
$$


If $\tau\left(1+\frac{n-2}{n} p\right) \leqq-n$, then we finish by applying (2.15) once more. Assuming that $\tau\left(1+\frac{n-2}{n} p\right)>-n$, we obtain from (2.15) (with $\tau$ replaced by $\left.\tau\left(1+\frac{n-2}{n} p\right)\right)$

$$
u(x)=O\left(|x|^{\left.r\left(1+\frac{n-2}{n} p\right) \frac{n-2}{n}\right)} \quad \text { at } \infty .\right.
$$

Substituting this back into $\tilde{K}$, and repeating this process, we conclude, after $k$ iterations, that either

$$
\begin{array}{ll}
K(x)=O\left(|x|^{\left[1+\frac{n-2}{n} p+\ldots+\left(\frac{n-2}{n} p\right)^{k+1}\right] \tau}\right) & \text { at } \infty \\
u(x)=O\left(|x|^{\left.\left[1+\frac{n-2}{n} p+\ldots+\left(\frac{n-2}{n} p\right)^{k+1}\right]\left(\frac{n-2}{n}\right) \tau\right)}\right. & \text { at } \infty,
\end{array}
$$

in case

$$
\left[1+\frac{n-2}{n} p+\ldots+\left(\frac{n-2}{n} p\right)^{k+1}\right] \tau>-n
$$

or otherwise $u(x)=O\left(|x|^{2-n}\right)$ at $\infty$.

Now, if $\frac{n-2}{n} p \geqq 1$, then eventually (2.21) fails to hold for large $k$ and therefore $u(x)=O\left(|x|^{2-n}\right)$ at $\infty$. If $\frac{n-2}{n} p<1$, then

$$
\left[1+\frac{n-2}{n} p+\ldots+\left(\frac{n-2}{n} p\right)^{k+1}\right] \tau \rightarrow \frac{n \tau}{n-(n-2) p}
$$

and (2.21) holds for all $k$ if and only if $p \leqq(n+\tau) /(n-2)$. Thus, if $p>$ $(n+\tau) /(n-2)$, then $u(x)=O\left(|x|^{2-n}\right)$ at $\infty$, and the remaining case of (2.18) follows from (2.20). Q.E.D.

Our main results are

Theorem 2.2. Every bounded positive entire solution $u$ of (2.14) with finite total mass must be radially symmetric about the origin and $u_{r}<0$ for $r>0$ provided that $K$ is radially symmetric, positive and decreasing for $r>0$ with $K(r)=O\left(r^{\tau}\right)$ near $\infty$ for some $\tau<0$, and that $p>(n+1+\tau) /(n-2)$. Furthermore, we have $u(r)=O\left(r^{2-n}\right)$ at $\infty$ and

$$
\lim _{r \rightarrow \infty} r^{n-2} u(r)=\frac{1}{n(n-2) \omega_{n}} \int_{\mathbb{R}^{n}} K u^{p} d x .
$$

Theorem 2.3. Suppose that $K$ satisfies the hypotheses in Theorem 2.2. If $p \geqq$ $(n+2) /(n-2)$ then every bounded positive entire solution of $(2.14)$ must have infinite total mass. 
Theorem 2.2 is a direct consequence of Lemma 2.3 and Theorem A.1 (in the Appendix below) while the limit in (2.22) is given by (7.1) in [NY2]. Theorem 2.3 may be proved as follows.

Proof of Theorem 2.3. Suppose for contradiction that $u$ is a bounded positive solution of (2.14) with finite total mass. Since $p \geqq(n+2) /(n-2)>$ $(n+1+\tau) /(n-2)$, we conclude from Theorem 2.2 that $u$ must be radially symmetric. Since all positive radial solutions have infinite total mass (by Theorem 6 in [NY2]), $u$ must also have infinite total mass. This contradicts the assumption on $u$. Q.E.D.

Remark 2.1. Theorems 1.2 and 1.3 are special cases of Theorems 2.2 and 2.3 $(n=3, \tau=-2)$.

\section{§3. Generalized Eddington Equation}

In this section we consider the following equation:

$$
\Delta u+H(x) f(u)=0 \quad \text { and } \quad u>0 \quad \text { in } \mathbb{R}^{n},
$$

where $f(0)>0$, as well as the corresponding ordinary differential equation in case $H(x)=H(|x|)$

$$
\begin{gathered}
u_{r r}+\frac{n-1}{r} u_{r}+H(r) f(u)=0 \quad \text { in } r>0, \\
u(0)=\alpha>0, \quad u_{r}(0)=0 .
\end{gathered}
$$

An important special class is the generalized Eddington equation where $f(u)=e^{2 u}$

and

$$
\Delta u+H(x) e^{2 u}=0 \quad \text { and } \quad u>0 \text { in } \mathbb{R}^{n},
$$

$$
\begin{gathered}
u_{r r}+\frac{n-1}{r} u_{r}+H(r) e^{2 u}=0 \quad \text { in } r>0, \\
u(0)=\alpha>0, \quad u_{r}(0)=0 .
\end{gathered}
$$

We shall assume throughout this section that $0 \neq H$ is locally Hölder-continuous on $\mathbb{R}^{n}$. (In fact, it is standard to see that our analysis below in the radial case applies to the case $H(r)=O\left(r^{\sigma}\right)$ at $r=0$ for some $\sigma>-2$. See, for instance, [N2], [NY2].) We will prove some general theorems of existence and nonexistence concerning (3.1) and (3.3), in particular, Theorems 1.4 and 1.5 will be established. Since we are interested only in positive solutions, we will use the convention $f(t)=f(0)$ for $t \leqq 0$. The following assumptions on $f$ will be used although not necessarily simultaneously,

$$
f \in C^{0,1}\left(\mathbb{R}, \mathbb{R}^{+}\right) \text {and } f(0)>0,
$$

$f$ is nondecreasing in $\mathbb{R}$,

$$
f^{q}(s) \text { is convex in } \mathbb{R} \text {, for some } 0<q<1 \text {. }
$$




\subsection{The Radial Case}

In this subsection we shall always assume that $H$ is a radial function. Denoting the (unique) solution of (3.2) by $u=u(r ; \alpha)$, we first improve the nonexistence result of [NY2] (Theorem D in the Introduction).

Theorem 3.1. Suppose that (3.5) and (3.6) hold and $H \geqq 0$. Then a necessary condition for the existence of a positive radial entire solution of (3.1) is

$$
\frac{1}{n-2} \int_{0}^{\infty} r H(r) d r<\int_{0}^{\infty} \frac{1}{f(t)} d t .
$$

Proof. For simplicity we first assume that $f \in C^{1}$. Suppose that $u$ is a positive radial entire solution of (3.1) with $u(0)=\alpha$. Then $u=u(r ; \alpha)$ satisfies (3.2) and is nonincreasing in $r>0$. Thus

$$
\frac{1}{f(u)}\left(r^{n-1} u_{r}\right)_{r}+r^{n-1} H(r)=0
$$

Integrating from 0 to $r$ and applying integration by parts to the first integral, we obtain

$$
\frac{u_{r}}{f(u)}+r^{1-n} \int_{0}^{r} \frac{s^{n-1}\left(u^{\prime}\right)^{2} f^{\prime}(u)}{f^{2}(u)} d s+r^{1-n} \int_{0}^{r} s^{n-1} H(s) d s=0
$$

in $r>0$. Now, integrating again over $[0, \infty)$ and changing the order of integration in the third term, we have

$$
\int_{0}^{\infty} \frac{u^{\prime}}{f(u)} d r+\int_{0}^{\infty} r^{1-n} \int_{0}^{r} \frac{s^{n-1} u^{\prime 2} f^{\prime}(u)}{f^{2}(u)} d s d r+\frac{1}{n-2} \int_{0}^{\infty} s H(s) d s=0 .
$$

Then (3.6) implies that

$$
\frac{1}{n-2} \int_{0}^{\infty} s H(s) d s<-\int_{0}^{\infty} \frac{u^{\prime}}{f(u)} d r=\int_{u(\infty)}^{\infty} \frac{d t}{f(t)}
$$

and (3.8) is proved. The general case $f \in C^{0.1}$ follows similarly. Q.E.D.

Corollary 3.1. The generalized Eddington equation (3.3) does not possess any positive radial entire solutions if

$$
\frac{1}{n-2} \int_{0}^{\infty} r H(r) d r \geqq \frac{1}{2}
$$

Observe that this improves Theorem $\mathrm{D}$ in the Introduction. Using the arguments (with obvious modifications) in the proof of Theorem 8.2 in [NY2], we obtain 
Theorem 3.2. Suppose that (3.5), (3.6) hold and $H \geqq 0$. If

$$
\frac{1}{n-2} \int_{0}^{\infty} r H(r) d r<\sup _{\alpha>0} \frac{\alpha}{f(\alpha)}
$$

then there exist $\alpha_{1}, \alpha_{2}$ with $0<\alpha_{1}<\alpha_{2}$ such that for every $\alpha \in\left[\alpha_{1}, \alpha_{2}\right] u(r ; \alpha)$ is a positive entire solution (which is bounded below by a positive constant) of (3.1).

Remarks. (i) From the proof of this theorem it is easily seen that if the number $\sup \alpha / f(\alpha)$ is assumed by some $\alpha *>0$, then in (3.10) the strict inequality " $<$ " may be replaced by an inequality "ㅇ to ensure the existence of at least one positive entire solution (i.e. $u\left(r ; \alpha^{*}\right)>0$ for all $r>0$ ). However, the positive lower bound of such a solution can no longer be guaranteed.

(ii) A argument like that in the proof of Theorem 8.3 in [NY2] also shows that under the condition (3.6) there exists $\alpha_{c}>0$ such that $u\left(r ; \alpha_{c}\right)$ is a positive entire solution of (3.1) with $u\left(r ; \alpha_{c}\right) \rightarrow 0$ as $r \rightarrow \infty$.

(iii) When $f(t)=e^{2 t}$, condition (3.10) becomes

$$
\frac{1}{n-2} \int_{0}^{\infty} r H(r) d r<\frac{1}{2 e} \text {. }
$$

Comparing this to (3.9) we see that a gap exists between the conditions for existence and nonexistence. The following example may prove to be useful in investigating this gap.

Assuming $H \geqq 0$ (thus $u_{r} \leqq 0$ ) and integrating (3.4), we have

$$
r^{n-1} u_{r}+e^{2 u(r)} \int_{0}^{r} s^{n-1} H(s) \leqq 0
$$

and

$$
e^{-2 u} u_{r}+r^{1-n} \int_{0}^{r} s^{n-1} H(s) d s \leqq 0 .
$$

Integrating again we obtain

$$
e^{2 u(r)} \leqq\left\{e^{-2 x}+\frac{1}{n-2} \int_{0}^{r}\left[1-\left(\frac{s}{r}\right)^{n-2}\right] s H(s) d s\right\}^{-1}
$$

where $\alpha=u(0)$. On the other hand, it is standard that (see e.g. [NY2])

$$
u(r)=\alpha-\frac{1}{n-2} \int_{0}^{r}\left[1-\left(\frac{s}{r}\right)^{n-2}\right] s H(s) e^{2 u(s)} d s .
$$

Thus

$$
u(r) \geqq \alpha-\frac{1}{n-2} \int_{0}^{r} s H(s)\left\{e^{-2 x}+\frac{2}{n-2} \int_{0}^{s}\left[1-\left(\frac{t}{s}\right)^{n-2}\right] t H(t) d t\right\}^{-1} d s .
$$


Set $H(r)=\frac{n-2}{m^{2}} \chi_{[0, m(1-\varepsilon)]}(r)$, where $m>0,0<\varepsilon<1$. Then

$$
\int_{0}^{s}\left[1-\left(\frac{t}{s}\right)^{n-2}\right] t H(t) d t=\frac{(n-2)^{2}}{2 n m^{2}} s^{2} \quad \text { for } 0<s<m(1-\varepsilon),
$$

and the right-hand side of (3.11) becomes for large $r$

$$
l(\alpha) \equiv \alpha-\frac{n}{2(n-2)} \log \left[1+\frac{n-2}{n}(1-\varepsilon)^{2} e^{2 \alpha}\right] .
$$

Computations shows that the maximum of $l$ in $\alpha>0$ is assumed at $\alpha=$ $\frac{1}{2} \log \frac{n}{2(1-\varepsilon)^{2}}$, and therefore $l(\alpha)$ is positive somewhere if and only if

$$
(1-\varepsilon)^{2}<\left(\frac{2}{n}\right)^{\frac{2}{n-2}} \equiv c_{n}
$$

Also notice that

$$
\frac{1}{n-2} \int_{0}^{\infty} r H(r) d r=\frac{1}{2}(1-\varepsilon)^{2}
$$

and $c_{n}<1$ with $c_{n} \rightarrow 1$ as $n \rightarrow \infty$. Thus we may first choose $\varepsilon$ appropriately and then modify $H$ so that it is smooth with $\frac{1}{n-2} \int_{0}^{\infty} r H(r) d r$ smaller than but arbitrarily close to $\frac{1}{2} c_{n}$. For such $H$, it follows from (3.11) that (3.4) possesses infinitely many positive entire solutions. Since $c_{n} \rightarrow 1$ as $n \rightarrow \infty$, this example indicates that (3.9) is almost best possible.

\subsection{The General Case}

This subsection is devoted to extending the results in subsection 3.1 to nonradial solutions. Our goal is to establish Theorems 1.4 and 1.5. The method we shall use to establish Theorem 1.4 is the barrier method (i.e. the super-solution and sub-solution method). The following result is a slight extension of Theorem 1.4 .

Theorem 3.3. Suppose that $H \geqq 0$ in $\mathbb{R}^{n}$ and that

$$
h \equiv \sup _{x \in \mathbb{R}^{n}} \int_{\mathbb{R}^{n}} \frac{H(y)}{n(n-2) \omega_{n}|x-y|^{n-2}} d y \leqq \frac{1}{2 e} ;
$$

then equation (3.3) possesses at least one positive entire solution in $\mathbb{R}^{n}$. Suppose that strict inequality in (3.14) holds, then (3.3) possesses infinitely many positive entire solutions in $\mathbb{R}^{n}$.

We first remark that the above result does admit an extension to general $H$ (without the positivity assumption) with a similar proof. Next, we observe that 
if $H \geqq 0$ is radial, then

$$
\sup _{x \in \mathbb{R}^{n}} \int_{\mathbb{R}^{n}} \frac{H(y)}{n(n-2) \omega_{n}|x-y|^{n-2}} d y=\int_{\mathbb{R}^{n}} \frac{H(y)}{n(n-2) \omega_{n}|y|^{n-2}} d y
$$

and therefore Theorem 3.3 reduces to Theorem 3.2 by Remarks (i) and (iii) in subsection 3.1. Finally we note that Theorem 3.3 also admits various extensions to the more general equation (3.1). We shall only include one such example here.

Corollary 3.2. Suppose that $H \geqq 0$ in $\mathbb{R}^{n}$ and that (3.5) and (3.6) hold. If

$$
\sup _{x \in \mathbb{R}^{n}} \int_{\mathbb{R}^{n}} \frac{H(y)}{n(n-2) \omega_{n}|x-y|^{n-2}} d y<\min \left\{\sup _{\alpha>0} \frac{\alpha}{f(\alpha)}, \infty\right\},
$$

then Equation (3.1) possesses at least one positive entire solution in $\mathbb{R}^{n}$.

To prove Theorem 3.3, we need the following lemma.

Lemma 3.1. Suppose that $H \geqq 0$ in $\mathbb{R}^{n}$ and that

$$
\phi(x) \equiv \int_{\mathbb{R}^{n}} \frac{H(y)}{n(n-2) \omega_{n}|x-y|^{n-2}} d y \in L^{\infty}\left(\mathbb{R}^{n}\right) .
$$

Then $\bar{\phi}(r) \rightarrow 0$ as $r \rightarrow \infty$.

Proof. It is standard to see (e.g. Lemma 2.8 in [LN]) that $\phi \geqq 0$ in $\mathbb{R}^{n}$ and that

Thus we have

$$
\Delta \phi+H=0 \quad \text { in } \mathbb{R}^{n} .
$$

$$
\begin{aligned}
& \bar{\phi}^{\prime \prime}+\frac{n-1}{r} \bar{\phi}^{\prime}+\bar{H}=0, \\
& \bar{\phi}^{\prime}(0)=0, \quad \bar{\phi}(0)=\phi(0)>0,
\end{aligned}
$$

where $\bar{\phi}, \bar{H}$ are defined by (1.15). Integrating (3.17) twice in the usual way and changing the order of integration, we obtain

Since

$$
\bar{\phi}(r)=\bar{\phi}(0)-\frac{1}{n-2} \int_{0}^{r} s \bar{H}(s) d s+\frac{1}{(n-2) r^{n-2}} \int_{0}^{r} s^{n-1} \bar{H}(s) d s .
$$

$$
\begin{aligned}
\bar{\phi}(0) & =\phi(0)=\int_{\mathrm{R}^{n}} \frac{H(y)}{n(n-2) \omega_{n}|y|^{n-2}} d y \\
& =\frac{1}{n-2} \int_{\mathrm{R}^{n}} \frac{\mid y ! \cdot H(y)}{n \omega_{n}|y|^{n-1}} d y \\
& =\frac{1}{n-2} \int_{0}^{\infty} r \bar{H}(r) d r
\end{aligned}
$$


we obtain

$$
\lim _{r \rightarrow \infty} \phi(r)=\lim _{r \rightarrow \infty} \frac{1}{n-2} \int_{0}^{\infty}\left(\frac{s}{r}\right)^{n-2} s \bar{H}(s) \chi_{[0, r]}(s) d s .
$$

Since $\left(\frac{s}{r}\right)^{n-2} s \bar{H}(s) \rightarrow 0$ as $r \rightarrow \infty$ and

$$
0 \leqq\left(\frac{s}{r}\right)^{n-2} s \bar{H}(s) \chi_{[0, r]}(s) \leqq s \bar{H}(s) \in L^{1}\left(\mathbb{R}^{+}\right),
$$

we conclude by the Lebesgue Dominated Convergence Theorem that $\bar{\phi}(r) \rightarrow 0$ as $r \rightarrow \infty$. Q.E.D.

We are now ready for the

Proof of Theorem 3.3. First, observe that $\log \frac{1}{2 e h} \geqq 0$ by (3.14). For every $0 \leqq a \leqq \frac{1}{2} \log \frac{1}{2 e h}$, we set $v(x) \equiv a$ in $\mathbb{R}^{n}$ and $w(x)=a+b \phi(x)$ where $\phi$ is given by (3.16) and $b=1 /(2 h)$. It is clear that $v$ is a strict sub-solution of (3.3) and $w \geqq v$ in $\mathbb{R}^{n}$. Since $2 a \leqq \log \frac{1}{2 e h}$, we have

$$
\Delta w+H e^{2 w} \leqq H\left(e^{2 a} e^{2 b \cdot t}-b\right)=H\left(e^{2 a}-\frac{1}{2 e h}\right) e \leqq 0
$$

and thus $w$ is a super-solution. This implies that (3.3) must have at least one solution $u$ with $v \leqq u \leqq w$ in $\mathbb{R}^{n}$ (see $e . g$. Theorem 2.10 in [N1]). Note that the strong maximum principle implies that $u>a$ in $\mathbb{R}^{n}$. Finally, it follows from Lemma 3.1 that $\bar{w}(r) \rightarrow a$ as $r \rightarrow \infty$. Therefore $\bar{u}(r) \rightarrow a$ as $r \rightarrow \infty$. This completes the proof. Q.E.D.

The proof of Corollary 3.2 goes much the same as that of Theorem 3.3 above, hence is omitted here.

We now conclude this section with the following extension of Theorem 1.5.

Theorem 3.4. Suppose that $H \geqq 0$ in $\mathbb{R}^{n}$ and that (3.5), (3.6) and (3.7) hold. If there exists a point $x_{0} \in \mathbb{R}^{n}$ such that

$$
\frac{1}{n-2} \int_{0}^{\infty} r \bar{H}_{x_{0}, p}(r) d r \geqq \int_{0}^{\infty} \frac{d t}{f(t)},
$$

where $p=1 / q$, then Equation (3.1) possesses no positive entire solution in $\mathbb{R}^{n}$.

Proof. Suppose for contradiction that $u$ is a positive entire solution of (3.1). Then

$$
\bar{u}_{x_{0}}^{\prime \prime}+\frac{n-1}{r} \bar{u}_{x_{0}}^{\prime}+\frac{1}{\omega_{n} r^{n-1}} \int_{\left|x-x_{0}\right|=r} H(x) f(u)=0 .
$$


Since

$$
\begin{aligned}
f^{q}\left(\bar{u}_{x_{0}}(r)\right) & =f^{q}\left(\frac{1}{\omega_{n}} \int_{|\xi|=1} u\left(x_{0}+r \xi\right)\right) \\
& \leqq \frac{1}{\omega_{n}} \int_{|\xi|=1} f^{q}\left(u\left(x_{0}+r \xi\right)\right) \\
& =\frac{1}{\omega_{n}} \int_{|\xi|=1}(H f(u))^{q} H^{-q} \\
& \leqq \frac{1}{\omega_{n}}\left(\int_{|\xi|=1} H f(u)\right)^{\frac{1}{p}}\left(\int_{|\xi|=1} H^{-\frac{1}{p-1}}\right)^{\frac{p-1}{p}},
\end{aligned}
$$

by Jensen's and Hölder's inequalities, we obtain

$$
\bar{H}_{x_{0}, p}(r) f\left(\bar{u}_{x_{0}}(r)\right) \leqq \frac{1}{\omega_{n}} \int_{|\xi|=1} H\left(x_{0}+r \xi\right) f\left(u\left(x_{0}+r \xi\right)\right) .
$$

Thus (3.19) becomes

$$
\bar{u}_{x_{0}}^{\prime \prime}+\frac{n-1}{r} \bar{u}_{x_{0}}^{\prime}+\bar{H}_{x_{0}, p}(r) f\left(\bar{u}_{x_{0}}(r)\right) \leqq 0 .
$$

Now the arguments in the proof of Theorem 3.1 show that

$$
\frac{1}{n-2} \int_{0}^{\infty} r H_{x_{0}, p}(r) d r \leqq \int_{\bar{u}_{x_{0}}(\infty)}^{\bar{u}_{x_{0}}(0)} \frac{d t}{f(t)}
$$

which contradicts (3.18). Q.E.D.

\section{Appendix. Symmetry of Positive Entire Solutions}

In [GNN], under mild conditions on $f(u)$ near $u=0$, it was established that a positive entire solution of the equation

$$
\Delta u+f(u)=0
$$

in $\mathbb{R}^{n}$ with suitable decay at $\infty$ must be radially symmetric (Theorems 1 and $1^{\prime}$ in [GNN]). An extension of this kind of result to cover the more general equation

$$
\Delta u+f(|x|, u)=0
$$

was also included there (Theorem $1^{\prime \prime}$ in [GNN]). In this appendix we intend to explore further in this direction. Making use of the decay of $f(|x|, u)$ at $x=\infty$, we are able to prove the same symmetry conclusion with minimal hypothesis on the decay of solutions of (A.1). For instance, we shall show that if $f(|x|, \cdot)$ decays faster than quadratically at $x=\infty$, then a (any) positive entire solution of (A.1) which tends to zero at $\infty$ must be radially symmetric. Moreover, in some cases of even faster decay of $f$ in $x$ at $\infty$, we shall prove that all bounded positive entire solutions of (A.1) are radially symmetric. In particular, we shall prove Propositions 1.1 and 1.2 in the Introduction. 
We first list the hypotheses on $f$ as follows.

(A.2) $f(r, t)$ is continuous in $r \geqq 0, t \geqq 0$, and $f(r, 0) \equiv 0$.

(A.3) $f(r, t)$ is nondecreasing in $t$, and strictly decreasing in $r$ for every $t>0$ fixed.

(A.4) There exist constants $p>0, \tau \leqq 0$ and $C>0$ such that

$$
0 \leqq f(r, t) \leqq C\left(1+r^{n}\right)^{\tau / n} t^{p}
$$

for all $r \geqq 0$ and $t \geqq 0$.

Our first observation is the following

Theorem A.1. Suppose that $u$ is a positive entire solution of the equation (A.1) with the decay $u(x)=O\left(|x|^{-m}\right)$ at $\infty$ for some $m>0$. If $f(r, t)$ satisfies (A.2), (A.3) and (A.4) in $[0, \infty) \times\left[0,\|u\|_{L^{\infty}}\left(\mathbb{R}^{n}\right)\right]$, and if $-\tau+p m>n+1$, then $u$ must be radially symmetric about the origin, and $u_{r}<0$ for $r>0$. Furthermore we have

$$
\lim _{r \rightarrow \infty} r^{n-2} u(r)=k>0 .
$$

Proof. From (A.4) and the decay of $u$ at $\infty$ we have

$$
f(|x|, u(x)) \leqq C|x|^{\tau-p m} \quad \text { at } \infty .
$$

Since $\tau-p m<-(n+1)$, it follows from the assumption $u(x) \rightarrow 0$ as $x \rightarrow \infty$ that

$$
u(x)=\int_{\mathbb{R}^{n}} \frac{f(|y|, u(y))}{c|x-y|^{n-2}} d y .
$$

(See, for instance, Lemma 2.2 and the first paragraph in the proof of Lemma 2.3.) Now the arguments in the proof of Theorem 1 in [GNN] can be carried over here with obvious minor modifications. We omit the details. Q.E.D.

The proof of Proposition 1.2 may be sketched as follows. Since $\tau<-(n+1)$ is assumed in (1.8), it is clear that $\lim _{x \rightarrow \infty} u(x)=u_{\infty}$ always exists and

$$
u(x)=u_{\infty}+\int_{\mathbb{R}^{n}} \frac{f(|y|, u(y))}{c|x-y|^{n-2}} d y
$$

(see e.g. Lemma 2.8 in [LN]). Then the arguments used above in the proof of Theorem A.1 yield the conclusion of Proposition 1.2. Note that $u_{\infty}$ needs not be zero here.

To prove Proposition 1.1, we first observe that (A.6) still holds with $u_{\infty}=0$ (by the hypothesis $\tau<-2$ ). (See e.g. Lemma 2.8 in [LN] again.) Next, by the arguments in the proof of Theorem 2.9 in [LN] we obtain the following

Lemma. Let $u$ be a bounded solution of (A.6) with $u_{\infty}=0$, and let $f$ satisfy (A.4) with $\tau<-2$. Then

provided that $p>(n+\tau) /(n-2)$.

$$
u(x)=O\left(|x|^{2-n}\right) \quad \text { at } x=\infty
$$


It is easily seen that Proposition 1.1 is a direct consequence of Theorem A.1 and the lemma above.

Acknowledgment. The research described above was supported in part by NSF grants DMS 8601246 and DMS 8801587.

Notes added in proof. We shall treat the case $1<p \leqq 2$, left open in Theorem 1.2, in a forthcoming paper.

\section{References}

[BFH] Batt, J., Faltenbacher, W., \& Horst, E., Stationary spherically symmetric models in stellar dynamics, Arch. Rational Mech. Anal. 93 (1986), 159-183.

[BL] Berestycki, H., \& Lions, P.-L., Nonlinear scalar field equations, I. Arch. Rational Mech. Anal. 82 (1983), 313-345.

[E] Eddington, A. S., The dynamics of a globular stellar system, Monthly Notices of the Royal Astronomical Society 75 (1915), 366-376.

[GNN] GIDAs, B., Nr, W.-M., \& NirENBERG, L., Symmetry of positive solutions of nonlinear elliptic equations in $\mathbb{R}^{n}$, Advances in Math., Supplementary Studies, 7A (1981), 369-402.

[K] KaWANO, N., On bounded entire solutions of semilinear elliptic equations, Hiroshima Math. J. 13 (1983), 125-158.

[K0] Kusano, T. \& OharU, S., Bounded entire solutions of second order semilinear elliptic equations with applications to a parabolic initial value problem, Indiana Univ. Math. J. 34 (1985), 85-95.

[LN] LI, Y., \& NI, W.-M., On conformal scalar curvature equations in $\mathbb{R}^{n}$, Duke Math. J. 57 (1988), 895-924.

[M] Matukuma, T., The Cosmos, Iwanami Shoten, Tokyo 1938.

[N1] NI, W.-M., On the elliptic equation $\Delta u+K(x) u^{(n+2) /(n-2)}=0$, its generalization and applications in geometry, Indiana Univ. Math. J. 31 (1982), 493-529.

[N2] NI, W.-M., Uniqueness, nonuniqueness and related questions of nonlinear elliptic and parabolic equations, Proc. Symp. Pure Math. 45 (1986) (F. Browder ed.), Part 2, 229-241.

[NY1] Ni, W.-M., \& Yotsutani, S., On Matukuma's equation and related topics, Proc. Japan Acad. (Series A) 62 (1986), 260-263.

[NY2] Ni, W.-M., \& YotsuTani, S., Semilinear elliptic equations of Matukuma-type and related topics, Japan J. Appl. Math. 5 (1988), 1-32. 